Check for updates

Cite this: Analyst, 2020, 145, 1943

\title{
Facile synthesis of a cyclodextrin-metal organic framework decorated with Ketjen Black and platinum nanoparticles and its application in the electrochemical detection of ofloxacin $\dagger$
}

\author{
Feng Luan, ${ }^{a}$ Yunfei Wang, ${ }^{a}$ Shuang Zhang, ${ }^{a}$ Xuming Zhuang, (D) *a Chunyuan Tian, ${ }^{a}$ \\ Xiuli Fu ${ }^{a}$ and Lingxin Chen (iD *b,c,d
}

\begin{abstract}
A facile and novel method was developed to improve the conductivity of a cyclodextrin-metal organic frameworks (CD-MOFs) by modifying it with Ketjen Black (KB) and platinum nanoparticles (PtNPs). The structural morphology and composition of this PtNPs/KB/CD-MOFs nanocomposite was characterized by transmission electron microscopy, scanning electron microscopy, Fourier transform infrared spectroscopy, X-ray photoelectron spectroscopy, elemental mapping, and Raman spectroscopy. The sensor was then evaluated for its ability to detect ofloxacin using cyclic voltammetry, and the results showed that the PtNPs/KB/CD-MOF had high sensitivity, satisfactory stability and good reproducibility. In addition, a great linear range was obtained with a concentration range of $0.08-100 \mu \mathrm{M}$ and a low detection limit of $0.037 \mu \mathrm{M}(\mathrm{S} / \mathrm{N}=3)$. Therefore, the prepared electrochemical sensor could be successfully used to detect ofloxacin in serum.
\end{abstract}

Received 19th December 2019,

Accepted 6th January 2020

DOI: $10.1039 / c 9 a n 02575 h$

rsc.li/analyst animals. ${ }^{6,7}$ Furthermore, ofloxacin has been found in the excreta of animals, which can cause environmental pollution. ${ }^{8,9}$ For this reason, it is necessary to monitor the content of ofloxacin in humans, animals and even in the environment. Some methods have been used to detect ofloxacin, such as mass spectrometry, fluorescence spectrometry, ${ }^{10,11}$ high-performance capillary electrophoresis, high-performance liquid chromatography, ${ }^{10}$ and electrochemical detection. ${ }^{12}$ Among these methods, the electrochemical detection method is an alternative method with the advantages of simple operation, low cost, and satisfactory sensitivity. In the electrochemical method, use of an appropriate electrode is the key factor for it being successful. Thus, it is a challenge to develop modified materials to improve the electrochemical performance of electrodes. ${ }^{13}$

Cyclodextrin-metal organic frameworks (CD-MOFs) have an abundance of network topological structures ${ }^{14}$ flexible honeycomb porous structures and high specific surface area. ${ }^{15,16}$ Since the organic ligands, metal ions or cluster particles can arrange in distinct directions, ${ }^{12}$ CD-MOFs can form different framework pore structures, thus exhibiting different adsorption, optical and electromagnetic properties. ${ }^{12}$ Hence, CD-MOFs can be used in electrochemical analyses because of their special structures and excellent performances. In addition, CD-MOFs can also be effectively combined with other active materials, which provide the possibility of using them as electrode materials. 
However, the disadvantage of CD-MOFs is that their electrical conductivity is not high, which hinders electron transfer on the electrode. Therefore, addition of conductive substances to CD-MOFs is an effective strategy to improve their electrical conductivity. ${ }^{17}$ According to reports, carbon-based materials such as Ketjen Black (KB), graphene, and carbon nanotubes ${ }^{18,19}$ generally have good electrical conductivity. Among them, KB has a high specific surface area and great stability and has satisfactory electrical conductivity. ${ }^{16}$ Therefore, KB can be added to enhance the conductivity of CD-MOFs when they are combined together.

In recent years, novel metal nanomaterials, including $\mathrm{Pt}, \mathrm{Au}$ and Pd, have been widely used in electrochemical sensors. ${ }^{20,21}$ Platinum nanoparticles (PtNPs) are a kind of functional nanomaterials $^{22}$ and generally refer to a suspension or colloidal form of particles with a submicron diameter of 2-20 nm dispersed in a liquid. The shapes of the PtNPs mainly include spheres, rods, cubes and squares. PtNPs have been used in numerous fields, such as physics, chemistry and medicine, for use in electrochemical sensors, catalytic reactions, fuel cells, optics, electronics and electromagnetism. ${ }^{16}$

In this work, CD-MOFs were modified with KB and PtNPs to fabricate a novel electrochemical sensor for the determination of ofloxacin. The results of the electrochemical measurements showed that the electrochemical sensor has high sensitivity and a low detection limit, with good stability and reproducibility. Therefore, the obtained novel PtNPs/KB/ CD-MOFs were evaluated for their reproducibility and selectivity, and were used for the determination of ofloxacin in serum.

\section{Experimental section}

\section{Reagents}

Beta-cyclodextrin ( $\beta$-CD), potassium hydroxide $(\mathrm{KOH})$, absolute ethanol $\left(\mathrm{C}_{2} \mathrm{H}_{5} \mathrm{OH}\right)$, chloroplatinic acid $\left(\mathrm{H}_{2} \mathrm{PtCl}_{4}\right), \mathrm{KB}$, potassium ferricyanide $\left(\mathrm{K}_{3}\left[\mathrm{Fe}(\mathrm{CN})_{6}\right]\right)$, potassium chloride $(\mathrm{KCl})$, sodium chloride ( $\mathrm{NaCl}$ ), ofloxacin, glucose, ascorbic acid (AA), and uric acid (UA) were obtained from Sinopharm Chemical Reagent Co. Ltd (Beijing, China, https:/www.sigmaaldrich. com/china-mainland.html). Fetal bovine serum (FBS, \#10439024) was purchased from Thermo Fisher Scientific (https://www.thermofisher.com/cn/zh/home.html), and it was stored at $-20{ }^{\circ} \mathrm{C}$ before use. All chemicals were of analytical grade without further treatment. Nanopure deionized and distilled water (DDW, 18.25 $\mathrm{M} \Omega \mathrm{cm}$ ) was used in all the experiments.

\section{Instrumentation}

Cyclic voltammetry (CV) and amperometry measurements were carried out on a CHI660C electrochemical workstation (Chenhua Instruments, Shanghai, China). A conventional three-electrode system was used to carry out all the electrochemical measurements, with a glassy carbon electrode (GCE) as the working electrode, platinum wire as the auxiliary elec- trode, and $\mathrm{Ag} / \mathrm{AgCl}$ electrode (in saturated $\mathrm{KCl}$ ) as the reference electrode. The microstructure and morphology of the practical samples could be obtained by scanning electron microscopy (SEM, S-4800) and transmission electron microscopy (TEM, JEOL 2010F, $200 \mathrm{kV}$ ). Fourier transform infrared spectroscopy (FTIR, Nicolet Magna 560) was also used to characterize the crystal structures and composition of the composite materials. The purity and elemental composition of the composite materials were further determined by X-ray photoelectron spectroscopy (XPS, ESCALAB 250xi), elemental mapping (APOLLO $\mathrm{X}$ ), and Raman spectroscopy (RENISHAW A-9570-2000). The crystallinity of the nanomaterials were characterized by X-ray diffraction (XRD, LabX XRD-6000). Nitrogen adsorption analyses were conducted by Brunauer-Emmett-Teller (BET) measurements (TriStarII 3020). All experiments were performed at room temperature.

\section{Preparation of the KB/CD-MOFs}

The KB/CD-MOFs were obtained following a previously reported procedure, ${ }^{23}$ that is, $2.4 \mathrm{~g}$ purified $\beta-\mathrm{CD}, 1 \mathrm{~g} \mathrm{KOH}$ and $0.010 \mathrm{~g}$ KB were added to $30 \mathrm{~mL}$ DDW/ethanol (4:6) solution. The mixture was stirred at room temperature for $30 \mathrm{~min}$ and then transferred to a reactor. The reactor was placed in an electric blast air drying box at $100{ }^{\circ} \mathrm{C}$ for three days and allowed to naturally cool to room temperature. The obtained white crystal was centrifuged for $10 \mathrm{~min}$ at $8000 \mathrm{rpm}$ and washed with a DDW/ethanol (4:6) solution. The final product was obtained by drying in an air dry oven. The CD-MOFs were prepared and treated under similar experimental conditions but without adding $\mathrm{KB}$.

\section{Preparation of the PtNPs}

PtNPs were synthesized as described in the ref. 24 and 25. $2 \mathrm{~mL} 2.5 \mathrm{mM} \mathrm{H}_{2} \mathrm{PtCl}_{4}$ and $50 \mu \mathrm{L} 0.1 \mathrm{M}$ sodium citrate solution were mixed, and then, DDW was added until reaching a total volume of $20 \mathrm{~mL}$. A sodium borohydride solution $(600 \mu \mathrm{L}$, $0.1 \mathrm{M}$ ) was added to the mixed solution and stirred vigorously. PtNPs are likely to be formed if the color of the solution became darker than its initial color. After stirring the solution for $1.5 \mathrm{~h}$ and naturally cooling it to room temperature, PtNPs were obtained.

\section{Fabrication of the modified electrodes}

Before the electrode was modified, the GCE was ground and polished on suede with 0.05 and $1 \mu \mathrm{m}$ alumina powder. Then, the electrode was sonicated in ethanol for two or three times and dried in air. The obtained KB/CD-MOFs and PtNPs were placed in an agate mortar with $5 \mathrm{~mL}$ ethanol, and the mixture was ground for $10 \mathrm{~min}$ before being sonicated. Then, $50 \mu \mathrm{L}$ of the mixture was transferred to a centrifuge tube with $10 \mu \mathrm{L}$ of Nafion, and the mixture was sonicated again. Finally, $5 \mu \mathrm{L}$ of the mixed product was dropped onto the polished GCE, and the GCE was placed under an infrared lamp to quickly dry the mixture. The GCE was denoted as PtNPs/KB/ CD-MOFs/GCE. 


\section{Results and discussion}

\section{Characterization of the CD-MOFs, KB/CD-MOFs, and PtNPs/ KB/CD-MOFs}

SEM and TEM were carried out to study the structural morphologies of the CD-MOFs, KB/CD-MOFs and PtNPs/KB/ CD-MOFs. Fig. 1A showed the features of the CD-MOFs, and its specific surface area was relatively large. The voids were slightly loose and evenly distributed, indicating that the $\mathrm{CD}-\mathrm{MOFs}$ could be classified as a porous material. The whole KB/CD-MOFs structure was shown in Fig. 1B, KB evenly distributed and closely connected with the CD-MOFs. In addition, the gaps were uniformly placed. The composite material of the KB/CD-MOFs was successfully synthesized. Fig. 1C showed that the PtNPs were distributed on the surface of the $\mathrm{KB}$ / CD-MOFs and mixed evenly and tightly. Therefore, the generated PtNPs/KB/CD-MOFs composite material was successfully synthesized and could be used in the following experiments. As shown in Fig. 1D, the uniform dispersed pore structure of the CD-MOFs could be clearly observed. The TEM image presented in Fig. 1E showed the skeleton structure of the $\mathrm{KB} /$ CD-MOFs, indicating that the KB and CD-MOFs were successfully combined. The KB was uniformly dispersed in the gaps of the CD-MOFs. However, there were still many gaps in the skeletal structure. Fig. 1F showed the morphology of the

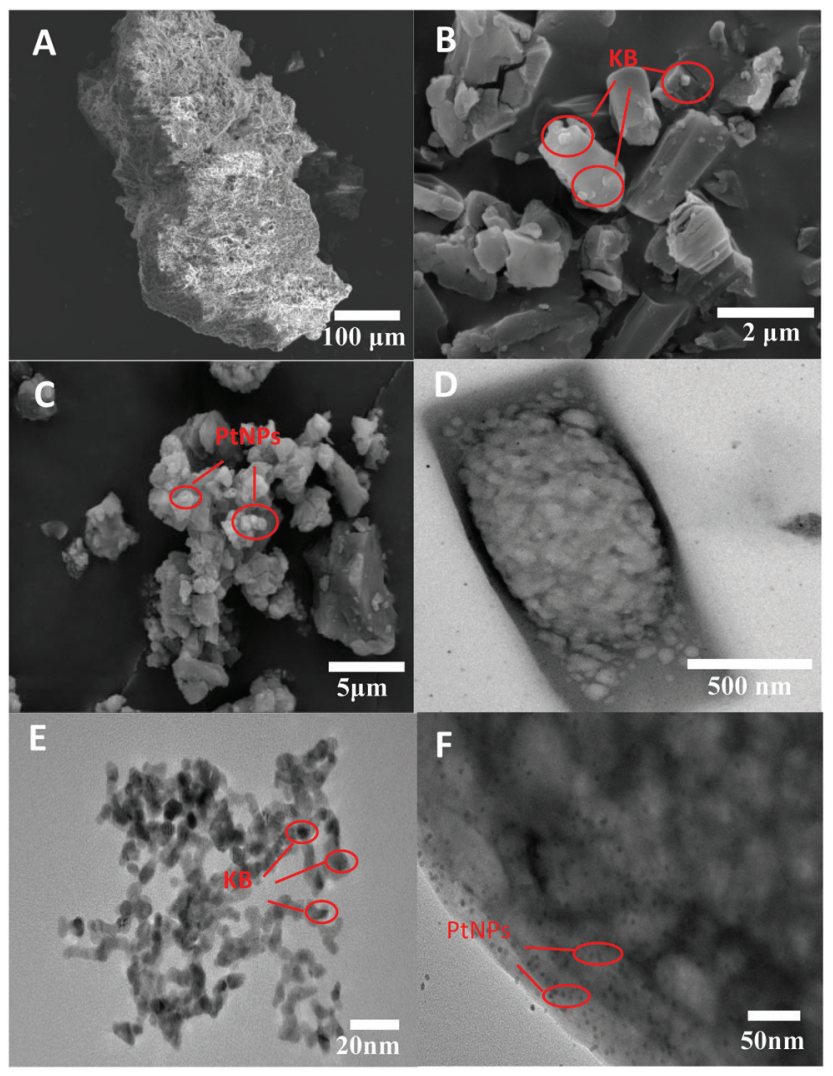

Fig. 1 SEM images of (A) the CD-MOFs, (B) KB/CD-MOFs, and (C) PtNPs/KB/CD-MOFs and TEM images of (D) the CD-MOFs, (E) KB/ CD-MOFs, and (F) PtNPs/KB/CD-MOFs.
PtNPs/KB/CD-MOFs. Compared with the KB/CD-MOFs, the PtNPs/KB/CD-MOFs were combined with PtNPs, causing the gaps to decrease. Therefore, it was confirmed that the $\mathrm{KB}$ and PtNPs both effectively combined with the CD-MOFs, implying that the PtNPs/KB/CD-MOFs were successfully synthesized.

The functional groups of the CD-MOFs, KB/CD-MOFs, and PtNPs/KB/CD-MOFs were analyzed by FTIR spectroscopy. As shown in Fig. 2A, the wide band at $3425 \mathrm{~cm}^{-1}$ was attributed to the stretching vibrations of the $-\mathrm{OH}$ group. The absorption peak at $1400 \mathrm{~cm}^{-1}$ was ascribed to the bending vibration in the $-\mathrm{OH}$ plane. In addition, the noticeable peak at $2980 \mathrm{~cm}^{-1}$ corresponded to the stretching vibrations of the $\mathrm{C}-\mathrm{C}$ in $\beta-\mathrm{CD}$. The absorption peak at $1642 \mathrm{~cm}^{-1}$ was due to the stretching vibration of $\mathrm{C}=\mathrm{C}$. Moreover, the peak at $1051 \mathrm{~cm}^{-1}$ was ascribed to the stretching vibration of the $\mathrm{C}=\mathrm{O}$ bond. Comparing the three FTIR spectra, the FTIR spectrum of the PtNPs/KB/CD-MOFs had the characteristic peaks of pure $\mathrm{KB} /$ CD-MOFs and CD-MOFs simultaneously, indicating successful synthesis of the nanomaterials.

Fig. 2B showed the Raman spectra of the synthesized PtNPs/KB/CD-MOFs nanoparticles. In the Raman spectrum, there was an obvious peak at approximately $440 \mathrm{~cm}^{-1}$, which could be caused by ethanol. In addition, the Raman bands at 1440 and $1580 \mathrm{~cm}^{-1}$ represented the D peak caused by the structural defects in $\mathrm{KB}$ and the $\mathrm{G}$ peak related to the firstorder scattering of the $\mathrm{E}_{2 \mathrm{~g}}$ phonons. ${ }^{26}$ In the Raman spectrum of the compound nanoparticle, the characteristic peaks could be used to indicate that the PtNPs/KB/CD-MOFs was successfully synthesized.

The XPS pattern of the PtNPs/KB/CD-MOFs was shown in Fig. 2C. The results demonstrated that there are three peaks at $70.15,287.79$ and $529.71 \mathrm{eV}$, which could be attributed to Pt 4f, $\mathrm{C} 1 \mathrm{~s}$ and $\mathrm{O} 1 \mathrm{~s}$, respectively. Besides, Fig. 2D showed the $\mathrm{C}$ 1s spectrum, and three kinds of carbon bonds were found:
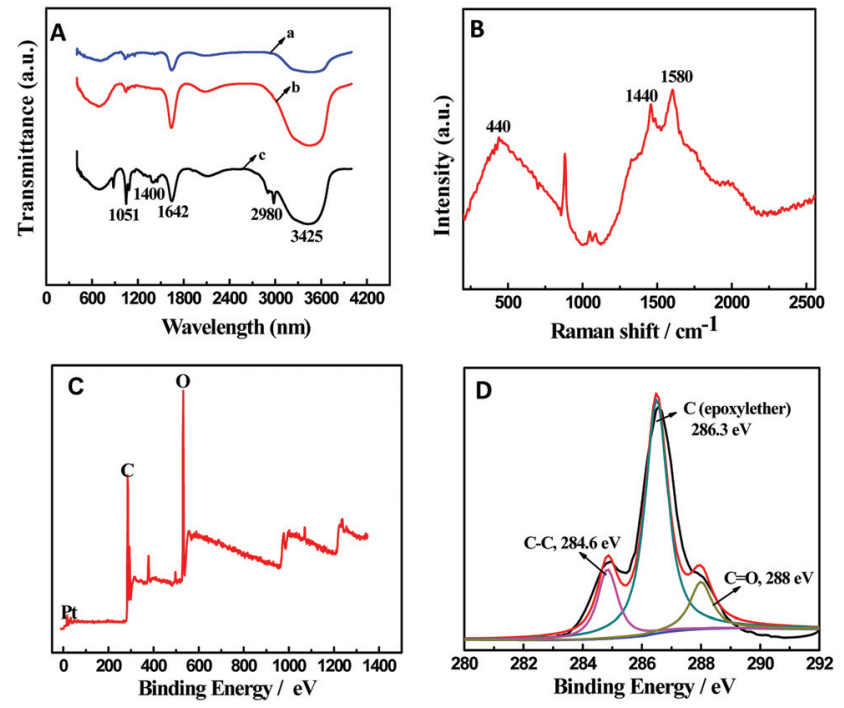

Fig. 2 (A) FTIR images of (a) the CD-MOFs, (b) KB/CD-MOFs, and (c) PtNPs/KB/CD-MOFs. (B) Raman and (C) XPS spectra of the PtNPs/KB/ CD-MOFs, and (D) high-resolution XPS spectra for $C 1$ s. 


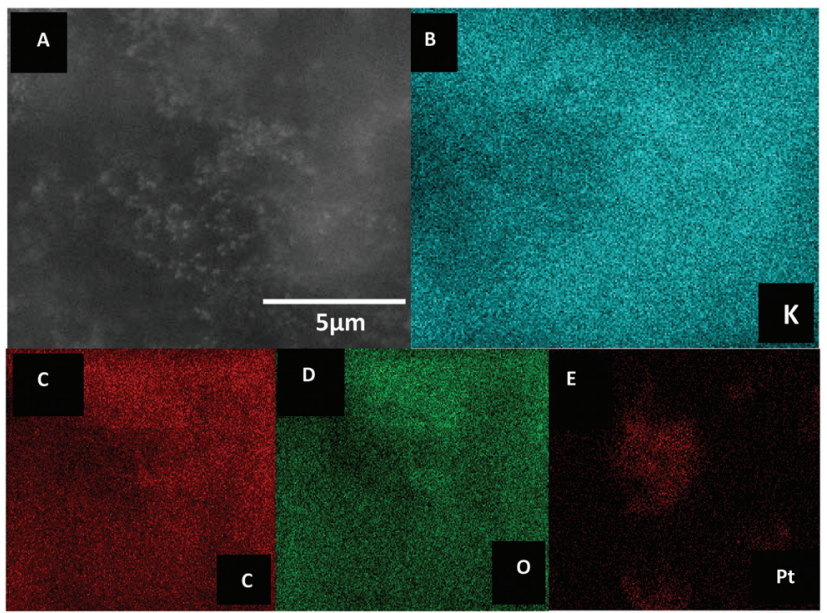

Fig. 3 EDS elemental mapping of $K(B), C(C), O(D)$, and $P t(E)$ performed on a sample area of the PtNPs/KB/CD-MOFs.

$\mathrm{C}-\mathrm{C}$ at $284.6 \mathrm{eV}, \mathrm{C}=\mathrm{O}$ at $288.0 \mathrm{eV}$, and epoxy-carbon at $287.7 \mathrm{eV}$. The above results demonstrated the presence of the PtNPs/KB/ CD-MOFs. In addition, SEM studies combined with elemental mapping were performed to verify the elemental composition of the PtNPs/KB/CD-MOFs. Fig. 3A-D showed elemental mapping for $\mathrm{K}, \mathrm{C}, \mathrm{O}$, and $\mathrm{Pt}$, respectively. The uniform distribution of $\mathrm{C}$ and $\mathrm{Pt}$ also highlights the incorporation and distribution of $\mathrm{KB}$ and PtNPs throughout the CD-MOF structure. Therefore, the SEM, TEM, FTIR spectroscopy, Raman spectroscopy, XPS, and elemental mapping results consistently confirmed the successful preparation of the PtNPs/KB/ CD-MOFs nanoparticles.

In addition, the crystallinity of the PtNPs/KB/CD-MOFs was studied, and the XRD pattern was shown in Fig. S2A. $\uparrow$ The characteristic diffraction peaks of $\mathrm{KB}$ and CD-MOFs were found at $2 \theta=7.2^{\circ}, 9.5^{\circ}, 12.9^{\circ}, 14.2^{\circ}$, and $16.6^{\circ}$, and the peak at $2 \theta=39.6^{\circ}$ could be assigned to the crystalline plane of $\mathrm{Pt}$, which corresponded to reports in the literature. ${ }^{27}$ The results showed that the PtNPs/KB/CD-MOFs were successfully synthesized.

Fig. $\mathrm{S} 2 \mathrm{~B} \uparrow$ showed the $\mathrm{N}_{2}$ adsorption-desorption of the PtNPs/KB/CD-MOFs that was measured with a liquid $\mathrm{N}_{2}$ bath. Before adsorption measurements, the samples were degassed in vacuum at $100{ }^{\circ} \mathrm{C}$ for $8 \mathrm{~h}$. The results showed that the surface area, pore volume and pore diameter were satisfactory.

\section{Optimizing the amount of KB}

The amount of $\mathrm{KB}$ used in the preparation of PtNPs/KB/ CD-MOFs affected the detection of ofloxacin. In this work, different amounts of $\mathrm{KB}(0.005,0.008,0.010,0.012,0.015 \mathrm{~g})$ were tested with a fixed concentration of PtNPs. As shown in Fig. S3A, $\uparrow$ the ordinate was the current difference before and after the addition of ofloxacin. The results revealed that the optimal amount of $\mathrm{KB}$ was found to be $0.010 \mathrm{~g}$ and that addition of too much or too little of $\mathrm{KB}$ had a detrimental effect on the detection of ofloxacin.

\section{Optimizing the amount of PtNPs}

In the process of preparing the PtNPs/KB/CD-MOFs, the amount of PtNPs also could affect the detection of ofloxacin. In this experiment, the effect of PtNPs was investigated by changing the amount from 0.5 to $9 \mathrm{~g}$ with $\mathrm{KB} / \mathrm{CD}-\mathrm{MOF}$ fixed at $3 \mathrm{~g}$; the result was shown in Fig. S3B. $\dagger$ It could be seen that the amount of $1 \mathrm{~g}$ PtNPs could get the best result on the detection of ofloxacin, that is, the mass ratio of $\mathrm{KB} / \mathrm{CD}-\mathrm{MOF}$ to PtNPs was $3: 1$. Hence, $1 \mathrm{~g}$ PtNPs was chosen as the most suitable amount.

\section{Conductivity of the PtNPs/KB/CD-MOFs}

The conductivity of the composite materials may play a dominant role in the electrochemical detection. Electrochemical impedance spectroscopy (EIS) was used to characterize the CD-MOFs, KB/CD-MOFs and PtNPs/KB/CD-MOFs. In the Nyquist plot of the impedance spectra shown in Fig. S3C, $\uparrow$ the semicircle portion at high frequencies could be attributed to the electron-transfer-limiting process, and the linear portion seen at low frequencies might correspond to diffusion. Besides, the increase in the diameter of the semicircle reflected the increase in the interfacial charge-transfer resistance $\left(R_{\mathrm{et}}\right)$. There was a substantially lower $R_{\mathrm{et}}$ of the $\mathrm{KB} /$ CD-MOF and PtNPs/KB/CD-MOF compared to that of the CD-MOF, indicating that after the encapsulation of the CD-MOF by $\mathrm{KB}$, the conductivity was higher. The conductivity of the CD-MOF might not be that large. After modification with PtNPs, the conductivity remained almost the same.

\section{CV behaviors on the differently modified electrodes}

The CV curves of the PtNPs/KB/CD-MOFs/GCE, KB/CD-MOFs/ GCE, CD-MOFs/GCE, and bare GCEs were carried out in $1 \mathrm{mM}$ $\mathrm{K}_{3}\left[\mathrm{Fe}(\mathrm{CN})_{6}\right]$ containing $0.1 \mathrm{M} \mathrm{KCl}$ at a scan rate of $100 \mathrm{mV} \mathrm{s}^{-1}$ to study the electron transfer of the different electrodes. The redox peaks of all the $\mathrm{CV}$ behaviors were clearly shown in Fig. 4A. Compared with the redox peak currents of the bare GCE, the currents of the CD-MOFs/GCE were higher, which could be attributed to the unique structure of the CD-MOFs that increased the specific surface area for electron transfer. Under the same conditions, the KB/CD-MOFs/GCE had a better current response than the CD-MOFs/GCE due to the addition of $\mathrm{KB}$, which greatly increased the conductivity of the material. However, the PtNPs/KB/CD-MOFs/GCE had the highest current response because of the excellent electrocatalytic properties and effective specific surface area of the PtNPs, so that the synthesized PtNPs/KB/CD-MOFs/GCE had the best electrochemical performance. The surface area of the electrodes was obtained according to the Randles-Sevcik formula, ${ }^{28,29}$

$$
I_{\mathrm{p}}=0.4463\left(F^{3} / R T\right)^{1 / 2} n^{3 / 2} A_{0} D_{0}^{1 / 2} c v^{1 / 2}
$$

where $I_{\mathrm{p}}$ is the anodic peak current, $R$ is the molar gas constant (8.314 $\mathrm{J} \mathrm{K}^{-1} \mathrm{~mol}^{-1}$ ), $F$ is Faraday's constant (96480 $\mathrm{C} \mathrm{mol}^{-1}$ ), $T$ is the temperature (298 K), $n$ refers to the number of electron transfer $(n=1), A_{0}$ is the surface area of the elec- 

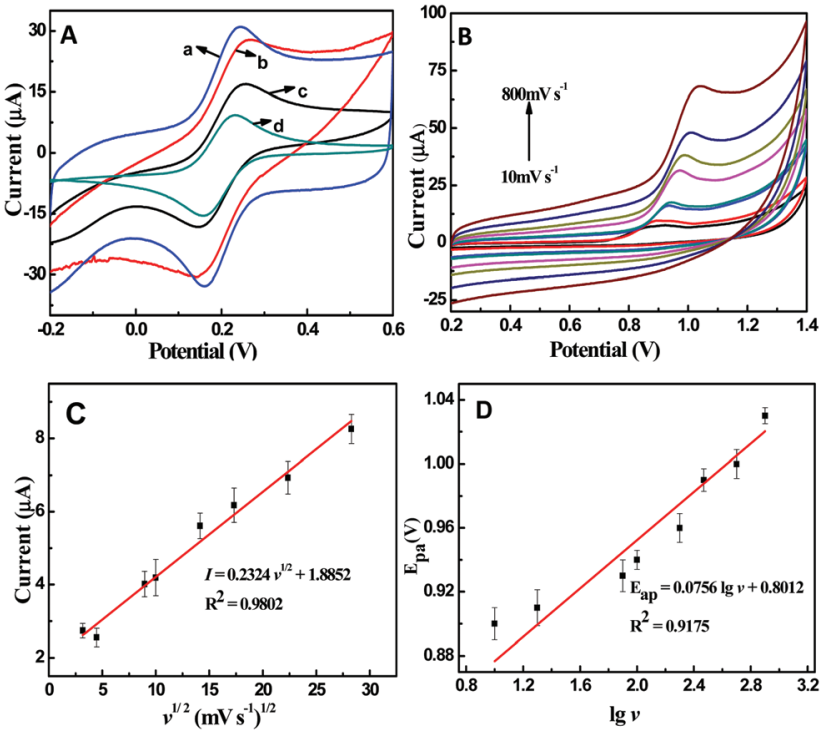

Fig. 4 (A) CV responses of (a) the PtNPs/KB/CD-MOFs/GCE, (b) KB/ CD-MOFs/GCE, (c) CD-MOFs/GCE, and (d) bare GCE in $\mathrm{K}_{3}\left[\mathrm{Fe}(\mathrm{CN})_{6}\right]$ containing $0.1 \mathrm{M} \mathrm{KCl}$. (B) $\mathrm{CV}$ responses of the PtNPs/KB/CD-MOFs/GCE in a $5 \mu \mathrm{M}$ ofloxacin solution with different scanning rates of $10,20,80,100$, $200,300,500$, and $800 \mathrm{mV} \mathrm{s}^{-1}$. (C) The linear relationship between the response current $\left(I_{\mathrm{pa}}\right)$ and the square root of the scan rate $\left(v^{1 / 2}\right)$. (D) The linear relationship between the peak potential $\left(E_{\mathrm{pa}}\right)$ and the logarithm of the scan rate $(\lg v)$.

trode $\left(\mathrm{cm}^{2}\right), D_{0}$ is the diffusion coefficient $\left(7.6 \times 10^{-6} \mathrm{~cm}^{2} \mathrm{~s}^{-1}\right)$, $c$ is the concentration of $\mathrm{K}_{3}\left[\mathrm{Fe}(\mathrm{CN})_{6}\right]\left(10^{-3} \mathrm{M}\right)$, and $v$ is the scan rate $\left(0.1 \mathrm{~V} \mathrm{~s}^{-1}\right)$. The surface areas of the bare GCE, CD-MOFs/GCE, KB/CD-MOFs/GCE, and PtNPs/KB/CD-MOFs/ GCE were calculated to be $0.048,0.085,0.140$, and $0.157 \mathrm{~cm}^{2}$, respectively. Therefore, the large surface area of the PtNPs/KB/ CD-MOF led to its great electrochemical performance.

\section{Effect of the scan rate}

To study the kinetics mechanism, CV curves of the PtNPs/KB/ CD-MOFs/GCE compound was obtained in PBS containing $5 \mu \mathrm{M}$ ofloxacin at different scan rates, and the results were shown in Fig. 4B. The scan rates were 10, 20, 80, 100, 200, 300, 500 , and $800 \mathrm{mV} \mathrm{s}^{-1}$. It was clear that all of the CV curves had a significant oxidation peak. In addition, the current response increased with an increasing scan rate. At the same time, there was a good linear relationship between the square root of the scan rate $\left(v^{1 / 2}\right)$ and the peak current $\left(I_{\mathrm{p}}\right)$, where, $I_{\mathrm{p}}=0.2324 v^{1 / 2}$ $+1.885\left(R^{2}=0.9802\right)$. It could be seen that the linear relationship was fine. Besides, Fig. $4 \mathrm{D}$ showed the linear relationship between the peak potential $\left(E_{\mathrm{pa}}\right)$ and the logarithm of the scan rate $(\lg v)$, which was $E_{\mathrm{pa}}=0.0756 \lg v+0.8012\left(R^{2}=0.9175\right)$. According to the Laviron equation: ${ }^{30}$

$$
E_{\mathrm{pa}}=E^{0}+(R T / \alpha n F) \ln \left(R T k_{\mathrm{s}} / \alpha n F\right)+(R T / \alpha n F) \ln v
$$

where $E^{0}$ is the formal redox potential; $T$ is the temperature $(298 \mathrm{~K}) ; R$ represents the molar gas constant $\left(8.314 \mathrm{~J} \mathrm{~K}^{-1}\right.$ $\left.\mathrm{mol}^{-1}\right) ; F$ represents Faraday's constant $\left(96480 \mathrm{C} \mathrm{mol}^{-1}\right) ; n$ is the number of electrons transferred in the processes controlled by diffusion; $\alpha$ represents the transfer coefficient, which can be assumed to be $0.5 ; k_{\mathrm{s}}$ refers to the standard rate constant of the reaction; and $v$ is the scan rate $\left(0.1 \mathrm{~V} \mathrm{~s}^{-1}\right)$. In conclusion, the number of electrons transferred $(n)$ in the processes controlled by diffusion was 1.77 . As a result, it was confirmed that the electrochemical oxidation of ofloxacin on the PtNPs/KB/ CD-MOFs/GCE should be a two-electron process. This conclusion was in accordance with the mechanism of ofloxacin in electrochemical reactions, as shown in Fig. S1.†

\section{Effect of pH}

To further investigate the effect of $\mathrm{pH}$ on the electrochemical response to ofloxacin, several CV curves were obtained in PBS containing ofloxacin $(5 \mu \mathrm{M})$ at different $\mathrm{pH}$ values, and the results were shown in Fig. 5A. The results showed a clear trend that with increasing $\mathrm{pH}$, the peak oxidation potential moved towards the negative direction. The $E_{\mathrm{p}}$-pH dependence of the ofloxacin reduction peak (Fig. 5B) was described with the following equation: $E_{\mathrm{p}}=-0.04331 \mathrm{pH}+1.324, R^{2}=0.9154$, which showed that the number of exchanged electrons and protons in the electrochemical reaction was the same according to the Nernst equation. This conclusion was also consistent with it had mentioned in the effect of the scan rate. Besides, the intensity of the ofloxacin reduction peak maxima was obtained at $\mathrm{pH}$ 7.4, therefore, $\mathrm{pH} 7.4$ was selected as the optimal value of PBS.

\section{Electrochemical sensing}

Fig. 6A showed the CV curves of PtNPs/KB/CD-MOFs/GCE in PBS containing ofloxacin at different concentrations $(0.08-100 \mu \mathrm{M})$. As it was shown, when the concentration of ofloxacin was $0.08 \mu \mathrm{M}$, the $\mathrm{CV}$ curve of the PtNPs/KB/ CD-MOFs/GCE had almost no oxidation peak. The current response significantly increased with the addition of ofloxacin, and the peak of oxidation was the highest when the concentration of ofloxacin reached $100 \mu \mathrm{M}$. This great electrochemical performance might be attributed to the large surface area and the excellent electrocatalytic properties of the PtNPs/KB/ CD-MOFs/GCE. In Fig. 6B, the linear relationship between the response current and the concentrations of ofloxacin was $I=$ $0.1463 c+1.9018\left(R^{2}=0.9901\right)$. In addition, the detection limit of ofloxacin was estimated to be $0.037 \mu \mathrm{M}(\mathrm{S} / \mathrm{N}=3)$. Comparing the detection limit and the linear range of the
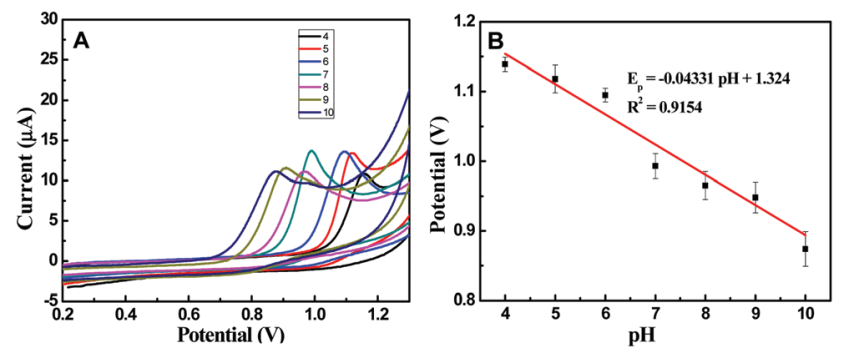

Fig. 5 (A) $C V$ responses of the PtNPs/KB/CD-MOFs in PBS containing ofloxacin $(5 \mu \mathrm{M})$ at different $\mathrm{pH}$ values (from 4 to 10). (B) The linear relationship between the potential $\left(E_{\mathrm{p}}\right)$ of ofloxacin and the $\mathrm{pH}$ of PBS. 

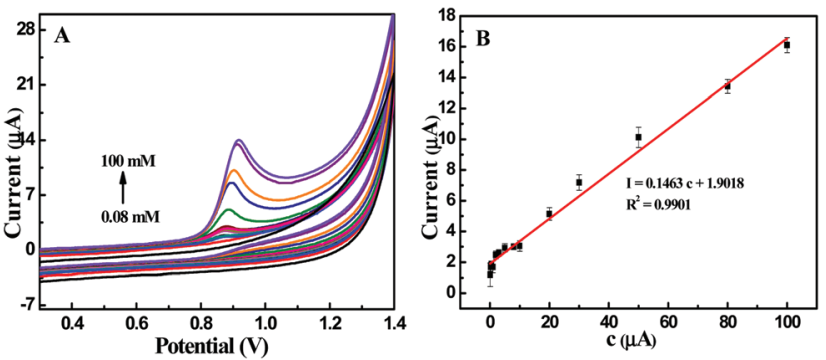

Fig. 6 (A) CV curves of the PtNPs/KB/CD-MOFs in PBS ( $\mathrm{pH}=7.4)$ containing different concentrations of ofloxacin from 0.08 to $100 \mathrm{mM}$. (B) The linear relationship between the response current $(l)$ and the ofloxacin concentrations (0.08-100 mM).

PtNPs/KB/CD-MOFs/GCE with that of other sensors (Table $\mathrm{S} 1 \dagger$ ), the PtNPs/KB/CD-MOFs/GCE electrochemical sensor could be used to effectively detect ofloxacin, and its analytical performance was excellent. In addition, the low detection limit of the PtNPs/KB/CD-MOFs/GCE electrochemical sensor was ascribed to its large specific surface area, good electrocatalytic properties of its active materials, and great electron transport properties.

\section{Reproducibility and selectivity}

In Fig. S4A, $\dagger$ the reproducibility of the PtNPs/KB/CD-MOFs/ GCE was further investigated. Six modified electrodes were prepared under the same conditions and used to detect ofloxacin in PBS. Besides, CVs were repeated six times on the same modified electrode. As shown in Fig. S4B, $\uparrow$ it was clear that all the CV curves had nearly coincident oxidation peaks, indicating the satisfactory reproducibility of the PtNPs/KB/CD-MOFs/ GCE.

The selectivity of the PtNPs/KB/CD-MOFs/GCE was assessed by adding some interfering substances, including glucose, $\mathrm{NaCl}, \mathrm{KCl}$ and UA, to the ofloxacin solution. In addition, some other substances found in blood samples were also tested, such as bovine serum albumin (BSA), methionine (Met), cysteine (Cys), lysine, $\mathrm{Ca}^{2+}, \mathrm{Mg}^{2+}$ and $\mathrm{Na}^{+}$. The results are shown in Fig. S5.† There were almost no changes in the CV response of ofloxacin before and after the addition of the interfering substances, and the relative errors $\left(E_{\mathrm{r}}\right)$ were less than $5 \%$, which indicated that the PtNPs/KB/CD-MOFs/GCE had a good anti-interference ability, which promoted its excellent selectivity for ofloxacin.

\section{Real sample analysis}

In order to test the practical application of PtNPs/KB/ CD-MOFs/GCE, the prepared sensor was used to detect ofloxacin in a serum using a standard addition method. CV curves for ofloxacin at concentrations of 10,50 , and $100 \mu \mathrm{M}$ were recorded. The results were shown in Table 1 . The obtained recoveries ranged from $91 \%$ to $103 \%$, which met the objective needs of the experiment. The above satisfactory results confirmed that the PtNPs/KB/CD-MOFs/GCE could be used for the detection of ofloxacin in a serum.
Table 1 Recoveries for the determination of ofloxacin in serum samples

\begin{tabular}{lccl}
\hline Spiked $(\mu \mathrm{M})$ & Found $(\mu \mathrm{M})$ & Recovery $(\%)$ & RSD $(\%)$ \\
\hline 10 & 9.16 & 91.60 & 0.92 \\
50 & 51.08 & 102.2 & 2.33 \\
100 & 99.20 & 99.20 & 1.62
\end{tabular}

\section{Conclusions}

In this study, a simple and sensitive electrochemical sensor based on the PtNPs/KB/CD-MOFs was developed for the determination of ofloxacin. The modified electrode was characterized by SEM, TEM, XPS, elemental mapping, FTIR spectroscopy, Raman spectroscopy, XRD, and CV. It was found that the sensor had excellent electrochemical performance, good reproducibility, high stability, and great selectivity, along with a low detection limit. The PtNPs/KB/CD-MOFs sensor was also used for the detection of ofloxacin in a serum. Therefore, this study was expected to be applicable to a wide range of electrochemical fields in the future.

\section{Conflicts of interest}

There are no conflicts of interest to declare.

\section{Acknowledgements}

This work was financially supported by the National Natural Science Foundation of China (21778047, 21675138, 21705139), National Natural Science Foundation of Shandong Province in China (ZR2017BB026) and Taishan Scholar Project Special Funding (No. ts20190962).

\section{Notes and references}

1 K. J. Huang, X. Liu, W. Z. Xie and H. X. Yuan, Microchim. Acta, 2008, 162, 227-233.

2 G. Carpini, F. Lucarelli and G. M. Mascini, Biosens. Bioelectron., 2005, 20, 167-175.

3 V. Andreu, C. Blasco and Y. Picó, TrAC, Trends Anal. Chem., 2007, 26, 534-556.

4 L. I. Ya-Li and X. U. Chuan-Lai, Food Sci., 2007, 28, 628633.

5 H. P. Sun, L. L. Cai, F. Q. Yan and L. I. Xia, Chin. J. Noso., 2008, 20, 77-84.

6 W. Ling, W. Ben, K. Xu, Y. Zhang, M. Yang and Z. Qiang, Chemosphere, 2017, 195, 252-256.

7 Z. Jiang, L. Qin, Y. Tang and M. Zhang, Electroanalysis, 2017, 29, 1-8.

8 A. Sangeeta and K. Do-Heyoung, Chem. Eng. J., 2018, 354, 692-705. 
9 Y. Xu, W. Yu, Q. Ma and H. Zhou, Sci. Total Environ., 2015, 530, 191-197.

10 F. A. El-Yazbi, Spectrosc. Lett., 1992, 25, 279-291.

11 Y. M. Issa, F. M. Abdel-Gawad, M. A. A. Table and H. M. Hussein, Anal. Lett., 1997, 30, 2071-2084.

12 M. Rizk, F. Belal, F. A. Aly and N. M. El-Enany, Talanta, 1998, 46, 83-89.

13 J. Davis, D. H. Vaughan and M. F. Cardosi, Enzyme Microb. Technol., 1995, 17, 1030-1035.

14 X. M. Lian, W. Zhao, X. L. Zhao and J. Solid, State Chem., 2013, 200, 265-270.

15 A. U. Czaja, N. Trukhan and U. Müller, Chem. Soc. Rev., 2009, 38, 1284-1280.

16 Y. Hua, L. I. Jun, H. Zhang, L. V. Ying and G. Shuang, Microporous Mesoporous Mater., 2014, 195, 87-91.

17 C. Wang, M. Zhou, Y. Y. Ma, H. Q. Tan, Y. H. Wang and Y. G. Li, Chem. - Asian J., 2018, 13, 2054-2059.

18 B. Jing and J. Xiue, Anal. Chem., 2013, 85, 80958101.

19 J. Li, J. D. Qiu, J. J. Xu, H. Y. Chen and X. H. Xia, Adv. Funct. Mater., 2010, 17, 1574-1580.
20 H. Shu, C. Gang, S. Jie, L. Cao, Q. Huang, Y. Zhang, T. Xia and Y. He, Sens. Actuators, B, 2015, 220, 331-339.

21 Y. Zhang, C. Gang, H. Shu, M. Oyama, L. Xiong and H. E. Yunbin, J. Power Sources, 2014, 262, 279-285.

22 X. U. Lihuan, Y. Zhu, X. Yang and L. I. Chunzhong, Mater. Sci. Eng., C, 2009, 29, 1306-1310.

23 J. J. Gassensmith, F. Hiroyasu, R. A. Smaldone, R. S. Forgan, Y. Y. Botros, O. M. Yaghi and S. J. Fraser, J. Am. Chem. Soc., 2011, 133, 15312-15315.

24 W. Yuan, J. Ren, D. Kai, L. Gui and Y. Tang, ChemInform, 2000, 31, 1622-1627.

25 Y. Li, W. Gao, L. Ci, C. Wang and P. M. Ajayan, Carbon, 2010, 48, 1124-1130.

26 G. Tao, M. Glerup, F. Krumeich, R. Nesper, P. Norby and J. Phys, Chem. C, 2008, 112, 13134-13140.

27 B. Panella and M. Hirscher, Adv. Mater., 2010, 17, 538-541.

28 Y. L. T. Ngo, T. H. Le, S. C. Jin and S. H. Hur, J. Alloys Compd., 2017, 712, 742-751.

29 I. Khan, U. J. Pandit, S. Wankar, R. Das and S. N. Limaye, Ionics, 2016, 23, 1-16.

30 Y. Wang, C. Li, T. Wu and X. Ye, Carbon, 2018, 129, 21-28. 